\title{
Peripheral neurectomies: A treatment option for trigeminal neuralgia in rural practice
}

\author{
Fareedi Mukram Ali, Prasant MC, Deepak Pai ${ }^{1}$, Vinit A Aher, Sanjay Kar², Safiya T \\ Department of Oral and Maxillofacial Surgery, SMBT Dental College and Hospital, Amrutnagar, Sangamner, ${ }^{1}$ Consultant Oral \& Maxillofacial \\ Surgeon, Sangamner; ${ }^{2}$ KIMSU, Karad, Maharashtra, India
}

\begin{abstract}
Background: Trigeminal neuralgia is a commonly diagnosed neurosensory disease of head, neck and face region, involving $5^{\text {th }}$ cranial nerve. Carbamazepine is the first line drug if there is decrease in efficacy or tolerability of medication, surgery needs to be considered. Factors such as pain relief, recurrence rates, morbidity and mortality rates should be taken in to account while considering which technique to use. Peripheral neurectomy is a safe and effective procedure for elderly patients and in rural and remote centers where neurosurgical facilities are not available. It is also effective in those patients who are reluctant for major neurosurgical procedures. Although loss of sensation along the branches of trigeminal nerve and recurrence rate are associated with peripheral neurectomy, we consider it as the safe and effective procedure in rural practice, which can be done under local anesthesia. Aims: The aim of this prospective study is to evaluate the long term efficacy of peripheral neurectomy with and without the placement of stainless steel screws in the foramina and to calculate the mean remission period after peripheral neurectomies for different branches of trigeminal nerve. Setting and Design: The sample was divided into 2 groups by selecting randomly the patients, satisfying inclusion criteria. Both groups were operated under local anesthesia by regional nerve blocks. In one group of patients after peripheral neurectomy, the proximal nerve stump was left alone in the foramina, and in another group of patients, obturation of foramina was done with stainless steel screws after peripheral neurectomy. Materials and Methods: Peripheral neurectomy was done on the terminal branches of trigeminal nerve in 14 patients. We selected only those cases that were experiencing pain after Carbamazepine therapy, all our patients were from rural and remote areas where facilities to neurosurgical centers are limited. Elderly patients who were unfit for surgical procedures and those patients who were reluctant for major neurosurgical treatments were considered for the study. Results: Post-surgical pain relief varied from 15 months to 24 months in cases where neurectomy was done without placing stainless steel screws in the foramina. Those cases where peripheral neurectomy was done along with the placement of stainless steel screws in the foramina, none of the patient had painful symptoms even after minimum 2 years of follow-up. Student's ' $t$ '-test of 2 groups showed the remission period to be statistically highly significant in patients with stainless steel screw obturation, having $P$-value $<0.0005$. Obturating the foramen with stainless steel screws can prevent nerve regeneration. Thus, remission of pain can be prolonged. Conclusion: Peripheral neurectomy is thus a safe and effective procedure for elderly patients, for those patients living in remote and rural places that cannot avail major neurosurgical facilities, and for those patients who are reluctant for major neurosurgical procedures.
\end{abstract}

Key words: Peripheral neurectomy, trigeminal neuralgia, therapy, peripheral nerves, surgery, India, rural health

\section{Introduction}

The term neuralgia is used to describe unexplained peripheral nerve pain, and the head and neck are the two most common sites of such neuralgias. ${ }^{[1]}$ In most

\begin{tabular}{|l|l|}
\hline \multicolumn{2}{|c|}{ Access this article online } \\
\hline Quick Response Code: & Website: \\
\hline & www.ruralneuropractice.com \\
\cline { 2 - 3 } & \\
\hline & \\
\hline
\end{tabular}

of the cases, a dentist sees the patient with the chief complaint of pain in the teeth and face before a physician does. ${ }^{[2]}$ Classic trigeminal neuralgia, as described by the International Headache Society, is 'Paroxysmal attacks of pain, lasting for a fraction of a second to 2 minutes, affecting one or more divisions of the trigeminal nerve' (Headache classification, subcommittee 2004). ${ }^{[3]}$ Trigeminal neuralgia is defined as sudden, usually unilateral, severe brief, stabbing recurrent episodes of pain within the distribution of one or more branches of the trigeminal nerve, which has profound effect on the quality of life. The mandibular branch of the trigeminal nerve is involved more commonly than

\section{Address for correspondence:}

Dr. Fareedi Mukram Ali, Department of Oral and Maxillofacial Surgery, SMBT Dental College and Hospital, Amrutnagar, Gulewadi Post, Sangamner, Ahmednagar Dist. Maharashtra, India. E-mail: faridi17@rediffmail.com 
the maxillary branch. ${ }^{[4]}$ This condition can be diagnosed from its characteristic clinical and symptomatic features. Trigeminal neuralgic patients present with lancinating type of pain along the involved branches, rather than pointing to a single tooth or a small area. This disease is usually seen in the middle aged or in elderly people.

Carbamazapine is the drug of choice in the management of trigeminal neuralgia. ${ }^{[5,6]}$ Peripheral nerve blocks with alcohol ${ }^{[7]}$ or lidocaine ${ }^{[8]}$ in the branches of trigeminal nerve; injecting botulinium toxin type $\mathrm{A}$ (Botox) ${ }^{[9]}$ into the trigger zone of trigeminal neuralgia are the means that have been tried recently for controlling pain. Trigeminal nerve block with high concentration lidocaine $(10 \%)$ is capable of achieving an intermediate period of pain relief, but only in those patients with low intensity and short duration of pain. ${ }^{[8]}$ Peripheral alcohol injections do produce short to medium term pain relief, but their role in the management of trigeminal neuralgia remains controversial because repeated alcohol injections can cause local tissue toxicity, inflammation and fibrosis..$^{[10]}$ The other main disadvantage of peripheral alcohol injections is the temporary weakness of the muscles of mastication due to close approximation of the motor root to the rest of the mandibular nerve. ${ }^{[11]}$ Furthermore, it can also cause the serious complication of burning alcohol neuritis. ${ }^{[10]}$ Partial pain relief is seen with botulinum toxin, which finally needs to be controlled with peripheral neurectomy. ${ }^{[9]}$

Peripheral neurectomy is a simple, low-risk procedure that can be done on all terminal branches of the 3 divisions of trigeminal nerve. Peripheral neurectomies were first tried in 1830; compilation and reviews were published by various authorities between 1869 and 1895. ${ }^{[11,12]}$ This procedure can be done in outpatient setting without general anesthesia. It is a post-ganglionic surgical operation, which involves an avulsion of the nerve after its exit from the cranium. ${ }^{[13]}$ It is an effective and safe treatment in rural areas, which lack the facilities for neurosurgical procedures, in elderly patients and in patients who are reluctant for major surgeries.

The aim of this prospective study is to evaluate the long term efficacy of peripheral neurectomy with and without the placement of stainless steel screws in the foramina and to calculate the mean remission period after peripheral neurectomies for different branches of trigeminal nerve.

\section{Materials and Methods}

The present study was conducted in the Dept of Oral and Maxillofacial Surgery, SMBT Dental College,
Sangamner Taluka, Maharashtra State, India; Patients were referred from various rural health centers and dental clinics, during the period of 2008-2009.

Diagnosis was made on the basis of history of classical display of paroxysmal pain, lasting for seconds to minutes, rapidly provokable along the distribution of branches of trigeminal nerve, triggered by light touch in a specific area or by eating, talking, washing the face, or by cleaning the teeth. The pain was described as intense, sharp, superficial, stabbing, or shooting - often like an electric shock. The pain was strictly unilateral at a particular paroxysm and was without any sensory or motor loss in the affected nerve area. A diagnostic nerve block of $2 \%$ lignocaine, with adrenaline 1:200,000 concentration gave complete relief from the symptoms.

Inclusion criteria were, all the patients being on carbamazepine, ranging from $600-1200 \mathrm{mg}$ /day who had become refractory to medical therapy. Only those patients who were not fit for major neurosurgery because of either an old age or co-morbidity, and those patients who were reluctant for major surgical procedures like microvascular decompression, trigeminal tractotomy were considered. Those cases with atypical presentation like bilateral involvements, uncommon distribution, longer than usual pain were excluded from the study. Panoramic radiograph was taken to rule out any odontogenic cause of the pain.

The sample was divided into 2-groups by selecting randomly the patients, satisfying inclusion criteria. Both the groups were operated under local anesthesia by regional nerve blocks of lidocaine $\mathrm{HCl} 2 \%$ with adrenaline 1:200000. In one group of patients after peripheral neurectomy, the proximal nerve stump was left alone in the foramina, and in another group of patients, obturation of foramina was done with stainless steel screws after peripheral neurectomy.

Peripheral neurectomies were done on mental nerve $(N=6)$, inferior alveolar nerve along with mental nerve $(N=2)$ and infraorbital nerve $(N=6)$. Peripheral neurectomies were done on infraorbital nerve without obturating the foramen in 3 patients, and with obturation of foramen with stainless steel screws in 3 patients. On mental nerve, peripheral neurectomies were done without obturating the foramen in 3 patients, and obturation of foramen with stainless steel screw in 3 patients.

The patients in our study were followed up at regular intervals for a minimum period of 2 years. Success of the surgery was defined accordingly: (i) Excellent (no pain, no medication), (ii) Good (no pain with medication), (iii) Poor (pain even after medication). 


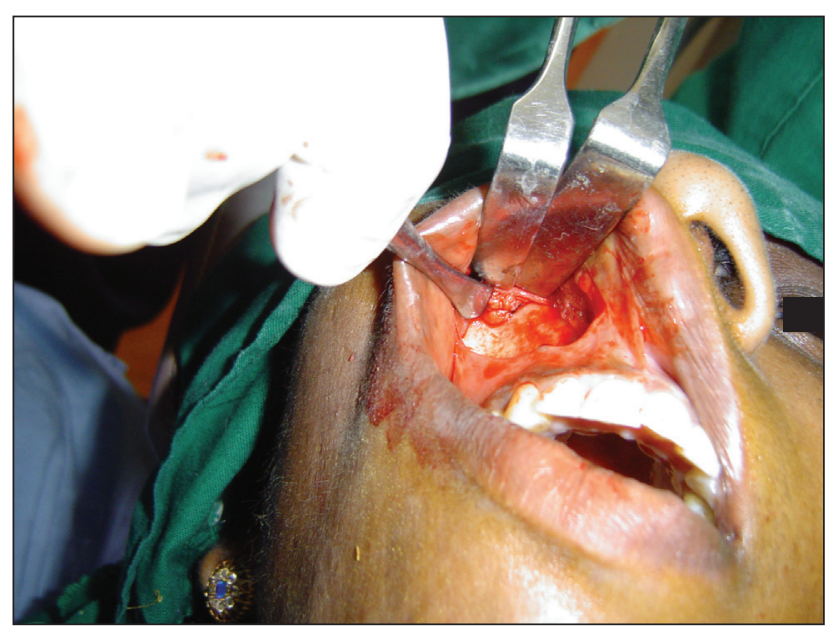

Figure 1: Exposure of the infraorbital nerve after vestibular incision

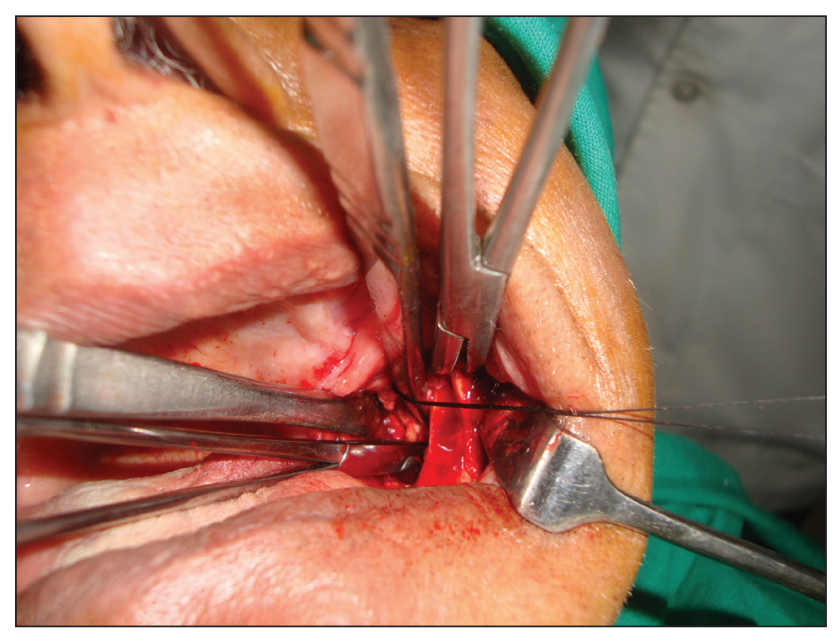

Figure 3: Ginwala approach, exposing the inferior alveolar nerve

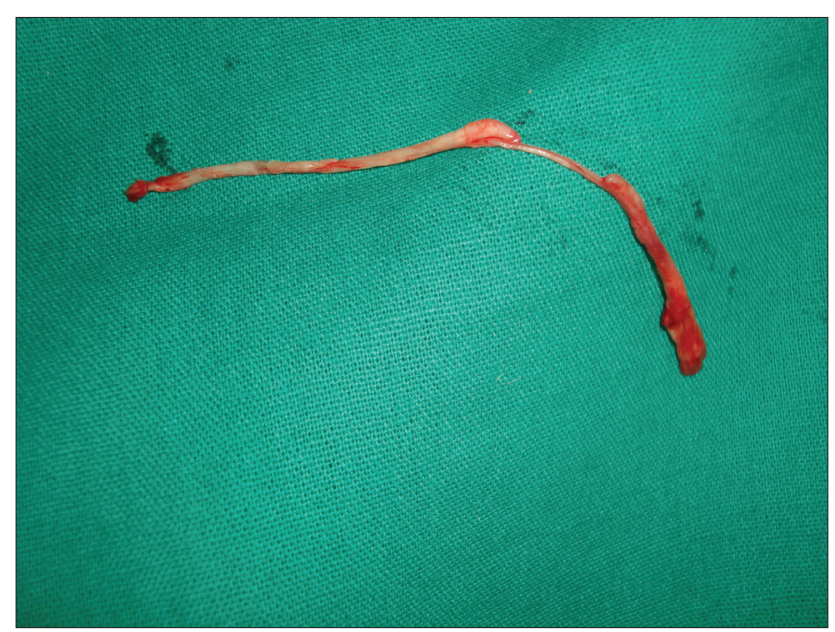

Figure 5: Avulsed nerve specimen

\section{Technique}

- Infraorbital neurectomy: Infraorbital neurectomy was performed through vestibular incision [Figure 1], dissection was carried out to expose the nerve. An infraorbital nerve was clamped and avulsed. The

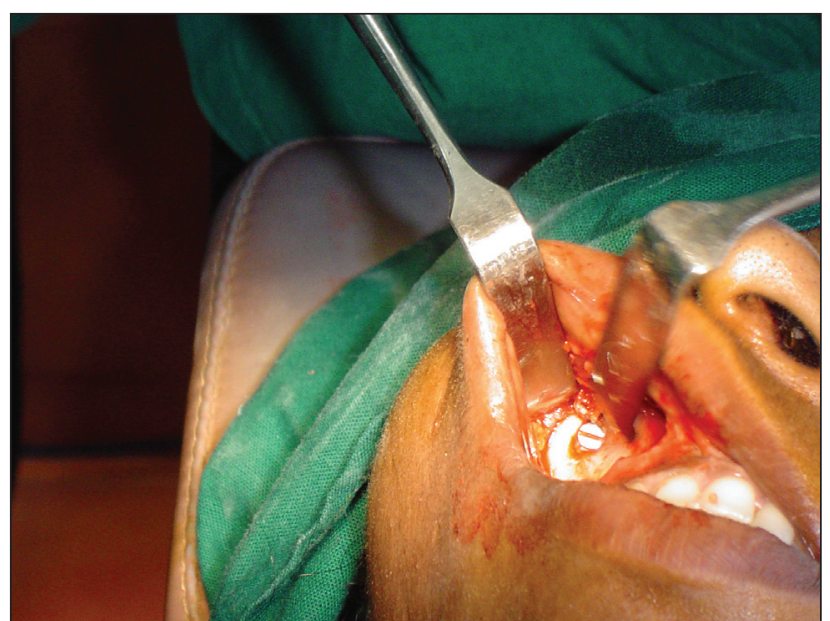

Figure 2: Postavulsion sealing of the infraorbital foramen using stainless steel screws

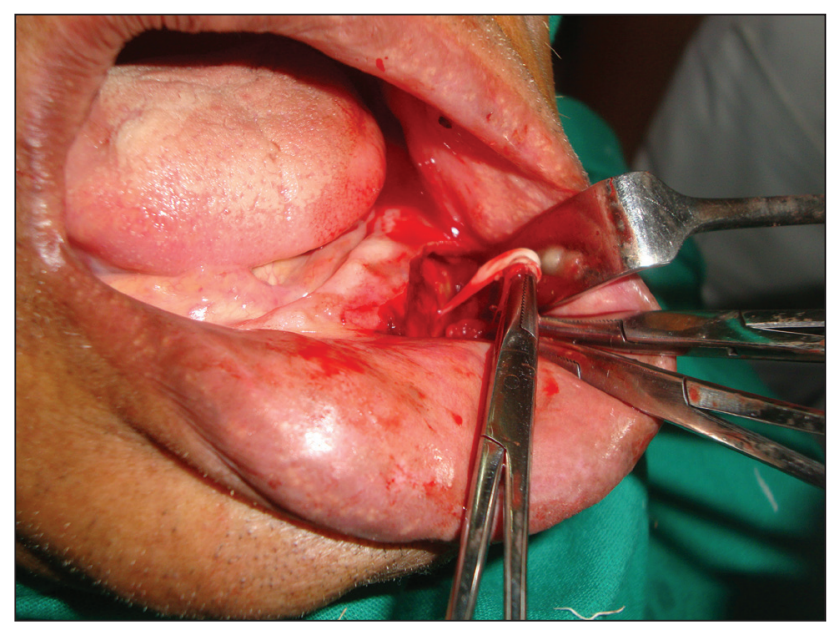

Figure 4: Exposure and avulsion of the mental nerve

orifice of the infraorbital foramen was then sealed by using a stainless steel screw [Figure 2].

- Mental nerve: The mucoperiosteal flap was raised using a crevicular incision along with a releasing incision in the premolar region [Figure 3].

- Inferior alveolar neurectomy: (Through Ginwala's incision $)^{[10]}$

An inverted Y-shaped incision was made along the anterior border of ascending ramus [Figure 4], which was then deepened on its medial aspect by means of a blunt and sharp dissection. The temporalis and medial pterygoid muscles were split, and the nerve was located, clamped and then cut below the instrument. Then the mental nerve was clamped at the mental foramen and avulsed [Figure 5].

\section{Results}

This Procedure was carried out in and around Sangamner Taluka during the period of 2008-2009. The study was 
conducted on 14 patients who fulfilled the inclusion criteria. Male: Female ratio was 4:10, age ranging from 40 to-72years. The infraorbital nerve was involved in 6 cases, inferior alveolar with mental nerve was involved in2 cases, and mental nerve was involved in 6 cases. Following neurectomy, pain was relieved in all the cases except two, in which it eventually subsided after 1 week. The patients were followed up on the $7^{\text {th }}$ post-operative day, and thereafter, reviewed regularly every month for a period of 2 years. Post-surgical pain relief varied from 15 months to 24 months in those cases where neurectomy was done without placing stainless steel screws in the foramina. In those cases where peripheral neurectomy was done with the placement of stainless steel screws in the foramina, none of the patient has painful symptoms till date even after follow-up of minimum 2 years. An assessment of the pain was done by visual analogue scale. In those cases where peripheral neurectomies were done on the inferior alveolar nerve along with mental nerve without obturating the foramina, a painfree period of about 15 to 19 months was followed by an early recurrence. On the other hand, the patients experienced a pain free period of about 17 to 24 months where neurectomies were done without obturating the foramen on the infraorbital nerve. Patients experienced a pain-free period of about 17 to 22 months where neurectomies were done without obturating the foramen on the mental nerve. Both the groups when compared statistically by student' $s$ ' $t$-test, it showed the ' $t$ ' value to be 5.56 with the difference of freedom (df) 12 , the $P$ value was found to be $<0.0005$; which suggests that the remission period in patients with obturation of foramina by screws is statistically highly significant. Success of the surgery was defined accordingly: (i) Excellent (no pain, no medication), (ii) Good (no pain with medication), (iii) Poor (pain even after medication). Excellent results were seen after 2 years in those cases where neurectomies were done along with the obturation of foramina with stainless steel screws $(n=6)$. In contrast, the cases where neurectomies were carried out without obturating with stainless steel screws, 5 showed good results, and 3showed poor results. There were no intra-operative or post-operative complications. None of the patients had post-operative infection.

\section{Discussion}

Carbamazepine, which was introduced in 1962, is still found to be the drug of choice in the management of trigeminal neuralgia. ${ }^{[5]}$ Response to carbamezepine treatment is, in part, diagnostic. ${ }^{[6]}$ Other drugs like gabapentine, pregabalines, dilantin sodium are also being used with good results. ${ }^{[14]}$ No patient should be considered for surgical treatment without the benefit of trying with carbamazepine for relief of pain. ${ }^{[4]}$ However, long term therapy with carbamazepine can lead to serious side effects such as, (i) development of resistance and intolerance, ii) drowsiness, (iii) vertigo, (iv) nausea. Serious consequences like (i) hemopoietic depression (ii) aplastic anemia (iii) abnormalities in liver functions. ${ }^{[4]}$

Currently available surgical options are, (1) Noninvasive technique: (a) peripheral neurectomy, (b) alcohol injections, (c) cryotherapy, (d) selective radio frequency thermocoagulation or, (2) Invasive technique: (i) open microvascular decompression, (ii) percutaneous: (a) radiofrequency rhizotomy, (b) retrogasserian glycerol rhizotomy, (c) balloon compression of trigeminal nerve, (d) stereostatic radiosurgery -gamma knife. ${ }^{[15]}$

Peripheral neurectomy was done for the first time in $18^{\text {th }}$ century with limited success. ${ }^{[11,12]}$ By dividing or avulsing a peripheral branch of the trigeminal nerve, the maxillofacial surgeon can achieve an exact, complete and long-lasting effect. Peripheral neurectomy can be done on the supraorbital and supratrochlear/ infratrochlear/ lacrimal nerves, the infraorbital nerve and the inferior alveolar, lingual and mental nerves. This surgery can be carried out as an outpatient procedure under local anesthesia in elderly and debilitated patients who are at an increased risk for undergoing invasive neurosurgical procedures. It is also useful in patients, reluctant for major neurosurgeries, and patients in rural places where facilities for advanced neurosurgical procedures are not available. As many patients and maxillofacial surgeons prefer this treatment owing to an advantage of minimum risk of morbidity, peripheral neurectomy has its role in the treatment of trigeminal neuralgia.

Peripheral neurectomies are definitely a better choice over peripheral nerve blocks with alcohol or phenol because the latter poses greater disadvantages like local tissue toxicity, inflammation, fibrosis and burning alcohol neuritis. ${ }^{[10]}$ Patients, who underwent both alcohol neurolysis and peripheral neurectomy, found the former painful and preferred the latter which gave more sure and durable pain relief. ${ }^{[16]}$ Partial pain relief was seen with botulinum toxin, which was finally controlled with peripheral neurectomy in case, reported by Ngeow and Nair. ${ }^{[9]}$ Zuniga et al ${ }^{[17]}$ assessed the clinical effects of botulinum toxin type A injections in 12 patients, with otherwise unresponsive idiopathic trigeminal neuralgia. Patients were infiltrated with 20-50 units of botulinum toxin in trigger zones. 10 of their patients reported a significant benefit from botulinum toxin injections, with reduction or even disappearance of pain, and remained pain-free for as long as 2 months. However, the cost of botulinum toxin is prohibitive in rural practice. 
The diagnosis in our study was based on a detailed history, clinical examination and control of pain by carbamazepine. All our patients were taking carbamazepine average $600-1200 \mathrm{mg} /$ day for 2-4 years and had become refractory to carbamazepine therapy.

Magnetic resonance imaging was not done in our study as the study was done in rural set-up, which lacks the advanced diagnostic imaging services and cost affordability was a factor too. Magnetic resonance imaging (MRI) may be useful in discovering underlying pathosis, associated with trigeminal neuralgia if patients have failed to respond to an initial conservative treatment. The patients, most likely to exhibit significant magnetic imaging resonance findings, are young and with pain in more than one trigeminal branch ${ }^{[18]}$ Further Study by Darlow et al. ${ }^{[19]}$ recommended the use of MRI only in patients in whom a trial of standard medications has been unsuccessful or in those who have atypical symptoms like (bilateral or uncommon distribution, longer than usual pain duration) to investigate whether demyelination, vascular compression or a tumor is the cause of the pain. Recent guidelines issued by the American Academy of Neurology (AAN) and European Federation of Neurological Societies (EFNS) have failed to find sufficient evidence to support or refute the fact that the presence of a neurocompression is the cause of TN..$^{[20,21]}$

A recent study done by Cerovic et al. ${ }^{[22]}$ reports that recurrence mainly appeared in the period between 12 to 15 months where peripheral neurectomies were done on the infraorbital nerve, and after 15 to 18 months where the neurectomies were done on the mandibular nerve. A report of pain relief from peripheral neurectomies, by Khanna and Galinde, ${ }^{[23]}$ for the infraorbital nerve is of 24 months and that for the mental nerve is of 26 months. Quinn ${ }^{[24]}$ reported a retrospective case series of 63 patients with 112 neurectomies, in which followup period of 0-9 years was noted, and a pain relief period of 24-32 months was reported. Grantham ${ }^{[25]}$ also reported an average pain relief period was 33.2 months in a case series of 55 patients who had undergone 55 neurectomies; follow-up was for 6 months to 8 years.

In comparison with the earlier studies, pain relief in our study was more or less similar. Post-surgical pain relief varied from 15 to 24 months in those cases where neurectomies were done without placing stainless steel screws in the foramina. In those cases where peripheral neurectomies were done on the inferior alveolar nerve along with mental nerve without obturating the foramina, recurrence of pain had occurred early after a pain-free period of about 15 to 19 months. On the other hand, patients experienced a pain-free period of about 17 to 24 months where neurectomies were done without obturating the foramen on the Infraorbital nerve.

Reviews on neurectomies and obturation of foramen with fat, ${ }^{[16]}$ titanium screws, ${ }^{[26]}$ gold foils, ${ }^{[27]}$ silicone ${ }^{[28]}$ are being published in oral and maxillofacial surgery literature since $60 \mathrm{yrs}$. Sung was one of the earliest authors who reported placing gold foil to obturate the foramina. ${ }^{[27]}$

The infraorbital foramen and mental foramen were sealed with stainless steel screws in 6 of the patients, following peripheral neurectomy. In those cases where peripheral neurectomies were done with the placement of stainless steel screws in the foramina, none of the patients from our study have presented with the symptoms of pain till date (at the end of 24 months). Regular follow-up is still being done with them.

The purpose of securing the foramina with a screw was to see whether there was change in the pain-free period. We are quite satisfied with those patients where neurectomies were done with the placement of stainless screws in the foramina. Hence, by preventing the nerve regeneration, the chances of recurrence in terms of time period is reduced. A similar study, done by Mason ${ }^{[18]}$ in (1972), achieved a success rate of $64 \%$ at the end of 12 months and $26 \%$ at the end of $4^{\text {th }}$ year. In a subgroup of 11 patients who underwent infra-orbital neurectomy, followed by occlusion of the canal, 7 patients were painfree at the end of $4^{\text {th }} \mathrm{yr}$. Hong-Sai ${ }^{[13]}$, in (1999), reported a case series of 12 patients with peripheral neurectomies, of which in 4 cases, the infraorbital foramen and mental foramen were obturated with titanium screws with no incidence of recurrence in a period of 4 years.

Freemont ${ }^{[16]}$ noted an average of 26.5 months of pain-free period in those cases where peripheral neurectomies were done and later obturated with fatty tissues. Obturation with fat gives good results due to good take-up of pedicled graft (fatty tissue), and fibrosis of the graft gave better obliteration of the foramen. In our study, obturation of the foramen was done by stainless steel screws instead of fatty tissue as the latter will cause distant site morbidity.

In those cases where peripheral neurectomies were done without obturating the foramina, the recurrence of pain was managed by carbamazepine therapy of $200 \mathrm{mg}, 12$ hourly. The response to carbamazepine therapy after recurrence to pain in those patients who had undergone peripheral neurectomies was better than before the treatment. This view was shared by many authors. ${ }^{[13,16,22,29]}$ 
In a country like India, where $70 \%$ of the population still belongs to the rural areas, peripheral neurectomies is still playing an important role in managing trigeminal neuralgia after more than 100 years. We are of the view that peripheral neurectomies offer temporary benefit although neurosurgery definitely has a role in the treatment of trigeminal neuralgia. But, every patient is not medically fit and may not be accessible to good neurosurgical facilities, either due to lack of infrastructure in rural places or due to financial affordability. Our views on peripheral neurectomies as a treatment alternative have been shared by many authors, Shah et al..$^{[29]}$ and Freemont and Millac. ${ }^{[16]}$

We observed that many patients in rural areas lead a poor quality of life. An isolation and depression is common among them because of associated excruciating pain. It is common for people suffering from trigeminal neuralgia to have poor oral hygiene along with other concomitant oral diseases because of the avoidance of tooth brushing. It is commonly observed in rural practice that, indiscriminate tooth extractions are done on the affected side without any relief from pain. Many of the patients were taking analgesics before a proper diagnosis could be done. In many of the cases, trigeminal neuralgia had been misdiagnosed by the general dental practitioners as TMJ disorder or sinusitis. All our patients were from rural background where insurance coverage is practically non-existing. Patient follow-up is a herculean task in rural setup because, as the symptoms subside, patients do not come for follow-up. Constant counseling is required. Also, many of these patients are reluctant to undergo advanced major surgical procedures in urban areas, owing to their low standard of living, high costs involved and the distances needed to be travelled. Peripheral neurectomy is a day care procedure that requires minimum armamentarium.

\section{Conclusion}

Peripheral neurectomy is a safe and effective procedure for elderly patients, for those patients living in remote and rural places that cannot avail major neurosurgical facilities, and for those patients who are reluctant or systemically contra-indicated for major neurosurgical procedures.

\section{References}

1. Shahrokh CB, Fariba F, Vincent JP. Diagnosis and treatment of patients with trigeminal neuralgia. J Am Dent Assoc 2004;135:1713-7.

2. Zakrzewska JM.Insights—facts and stories behind trigeminal neuralgia. Gainesville, FL: Trigeminal Neuralgia Association; 2006.

3. Headache Classification Subcommittee: The international Classification of headache. Disorders. $2^{\text {nd }}$ ed.Cephalalgia 2004;24:16-21.
4. Loh HS, Ling SY, Shanmuhasuntharam P, Zain R, Yeo JF, Khoo SP. Trigeminal neuralgia- a retrospective survey of a sample of patients in Singapore and Malaysia. Aust DentJ1998;43:188-91.

5. McQuay H, Carroll D, Jadad AR, Wiffen P, Moore A. Anticonvulsant drugs for the management of pain: A systemic review. Br Med J 1995; 311:1047-52.

6. Fields HL. Treatment of trigeminal neuralgia. N Engl J Med 1996;334: $1125-6$.

7. Han KR, Kim C. Brief report: The long-term outcome of Mandibular nerve block with alcohol for the treatment of trigeminal neuralgia. Anesth Analg 2010;111:550-3.

8. Han KR, Kim C, Chae YJ, Kim DW. Efficacy and safety of high concentration lidocaine for trigeminal nerve block in patients with trigeminal neuralgia. Int J Clin Pract 2008;62:248-54.

9. Ngeow WC, Nair R. Injection of botulinum toxin type A (Botox) into trigger zone of trigeminal neuralgia as a means to control pain. Oral Surg Oral Med Oral pathol Oral Radiol Endodtol 2010;109:e47-50.

10. Malik N. Text Book of Oral and Maxillofacial Surgery. New Delhi, India: JP Brother Medical Publishers; 2008.p. 685-97.

11. Wilkins RH. Historical overview of surgical techniques for trigeminal neuralgia. Tech Neurosurg 1999;5:202-17.

12. Fowler GR. The operative treatment of facial neuralgia: A comparison of methods and results. Ann Surg 1886;3:269-320.

13. Hong-Sai L. Surgical treatment of trigeminal Neuralgia. J Oral Rehab1999;26:613-7.

14. Zakrzewska JM. Facial Pain: Neurological and non-neurological. J Neurol Neurosurg Psychiatry 2002;72 Suppl:ii 27-32.

15. Bagheri S, Farhidvash F, Perciaccante V. Diagnosis and treatment of patients with trigeminal neuralgia. J Am Dent Assoc 2004;135:1713-7.

16. Freemont AJ, Millac P. The place of peripheral neurectomy in the management of trigeminal neuralgia. Postgrad Med J 1981;57:75-6.

17. ZunigaC, Díaz S, Piedimonte F, Micheli F. Beneficial effects of botulinum toxin type A in trigeminal neuralgia. Arg Neuropsiquiatr 2008;66:500-3.

18. Yang J, Simonson TM, Ruprecht A, Meng D, Vincent SD, Yuh WT. Magnetic resonance imaging used to assess patients with trigeminal neuralgia. Oral Surg Oral Med Oral Pathol Oral Radiol Endodontol 1996;81:343-50.

19. Darlow LA, Brooks ML, Quinn PD. Magnetic Resonance imaging in the diagnosis of trigeminal neuralgia. J Oral Maxillofac Surg 1992;50:621-6.

20. Cruccu G, Gronseth G, Alksne J, Argoff C, Brainin M, Burchiel K, et al. AAN-EFNS guidelines on trigeminal neuralgia management. Eur J Neurol 2008;15:1013-28.

21. Gronseth G, Cruccu G, Alksne J, Argoff C, Brainin M, Burchiel K,et al. Practice Parameter: The diagnostic evaluation and treatment of trigeminal neuralgia (an evidence based review): Report of the quality Standards Subcommittee of the American Academy of Neurology and the European federation of Neurological Societies. Neurology 2008;71:1183-90.

22. Cerovic R, Juretic M,Gobic MB. Neurectomy of the trigeminal nerve branch: Clinical evaluation of an "obsolete" treatment. J CranioMaxillofac Surg 2009;37:388-91.

23. Khanna JN, Galinde JS. Trigeminal neuralgia report of 140 cases. Int J Oral Surg 1985;14:325-32.

24. Quinn J H. Repetitive peripheral neurectomies for neuralgia of $2^{\text {nd }}$ and $3^{\text {rd }}$ divisions of the trigeminal nerve. J Oral Surg 1965;23:600.

25. Grantham E, Segerberg LH. An evaluation of palliative surgical procedures in trigeminal neuralgia. J Neurosurg 1952;9:390.

26. Mason DA. Peripheral neurectomy in the treatment of trigeminal neuralgia of second and third divisions. J Oral Surg 1972;30:113-20.

27. Sung RR. Peripheral neurectomy as treatment for incipient trigeminal neuralgia. Oral Surg Oral Med Oral Pathol 1951;4:296-302.

28. Cogan MI. Evaluation of silicone rubber in preventing inferior alveolar nerve regeneration. J Oral Surg1968;26:99.

29. Shah SA, Khattak A, Shah FA, Khan Z. The role of peripheral neurectomies in the treatment of trigeminal neuralgia in modern practice. Pak Oral Dent J 2008;28:237-40.

How to cite this article: Ali FM, Prasant MC, Pai D, Aher VA, Kar S, Safiya T. Peripheral neurectomies: A treatment option for trigeminal neuralgia in rural practice. J Neurosci Rural Pract 2012;3:152-7.

Source of Support: Nil. Conflict of Interest: None declared. 